\title{
Existence of self-similar evolution of crystals grown from supersaturated vapor
}

\author{
YOSHIKAZU GIGA ${ }^{\dagger}$ \\ Department of Mathematics, Hokkaido University, \\ Sapporo 060-0810, Japan \\ AND \\ PIOTR RYBKA ‡ \\ Institute of Applied Mathematics and Mechanics, Warsaw University, \\ Banacha 2, 02-097 Warszawa, Poland
}

[Received 5 September 2003 and in revised form 2 June 2004]

\begin{abstract}
We study a cylindrical crystalline flow in three dimensions coupled to a diffusion field. This system arises in modeling crystals grown from supersaturated vapor. We show existence of self-similar solutions to this system under a special choice of interfacial energy and kinetic coefficients.
\end{abstract}

\section{Introduction}

In [GR1] we considered a model of crystals grown from supersaturated vapor. We established existence of solutions to the evolution equations. Here we want to construct a special kind of maximal solutions, namely a self-similar motion.

Our work was motivated by experiments. Namely, in their laboratory Gonda and Gomi [GoG] have grown specimens of elongated prisms of ice crystals similar to those formed in the atmosphere and found mostly in Antarctica. Theoretical foundation for our work has been laid down by Seeger [Se] who studied planar polygonal crystals. Later his approach was extended to three dimensions by Kuroda et al. [KIO]. These papers, however, do not include the Gibbs-Thomson relation on the free boundary. We think that it is important to include this effect, especially for small crystals.

In [GR1] and here we make a simplifying assumption that the evolving crystal is a (right circular) cylinder $\Omega(t)$, and not a hexagonal prism. This approach is in fact quite frequent in the physics literature (see [Ne], [YSF]). We shall study solely the evolution of such right cylinders. The description of the evolving interface is then relatively easy. Namely, it requires specifying the radius $R$ and the height $2 L$.

We now recall the equations of motion which we studied in [GR1]. The first one, for the supersaturation $\sigma$ outside the crystal $\Omega(t)$, is

$$
\Delta \sigma=0 \quad \text { in } \mathbb{R}^{3} \backslash \Omega(t) .
$$

It means that the mass is transported by diffusion and this transport is much faster than the motion of the free boundary $\partial \Omega(t)$, hence the term $\partial \sigma / \partial t$ on the right-hand side of $(1.1)$ is dropped. It is

\footnotetext{
† Present address: Graduate School of Mathematical Sciences, University of Tokyo, Komaba 3-8-1, Tokyo 153-8914, Japan.

E-mail: rybka@hydra.mimuw.edu.pl
} 
also physically reasonable to assume that $\sigma$ has a specific value at infinity, i.e.

$$
\lim _{|x| \rightarrow \infty} \sigma(t, x)=\sigma^{\infty} .
$$

We now explain the next equation representing the mass conservation on a crystal surface. It is a Stefan-type condition. The evolution model we present is meant not only for crystals grown from vapor but also for those growing from solution. Since the presentation is simpler for the latter we explain the equation of mass conservation for crystals grown from solution. In this case $\sigma$ is given as

$$
\sigma=\frac{C-C_{e}}{C_{e}},
$$

where $C=C(t, x)$ represents the concentration of atoms and $C_{e}$ is the saturated concentration which is a constant independent of $x$ and $t$. The velocity $V$ of the growing crystal is determined by the normal derivative of $C$ on the surface,

$$
v_{c} D \frac{\partial C}{\partial \mathbf{n}}=V \quad \text { on } \partial \Omega(t)
$$

where $\mathbf{n}$ is the outer normal. Here $v_{c}$ represents the volume of an atom of the crystal and $D>0$ is the diffusion coefficient of atoms in the solution. This equation expresses the mass conservation. By normalizing the time variable we may assume that this Stefan-like mass conservation is of the form

$$
\frac{\partial \sigma}{\partial \mathbf{n}}=V \quad \text { on } \partial \Omega(t)
$$

Here $V$ is assumed to be constant on each facet $S_{i} \subset \partial \Omega(t), i=T, B, \Lambda$, i.e. on the top, bottom and lateral surface. The notation we use is explained in Section 2.

The hypothesis that $V$ is spatially constant on each $S_{i}$ relates to stability properties of facets or facet non-breaking properties. This property is not always natural, especially when $\Omega(t)$ is large (see [GiGi], [BNP1], [BNP2]). We do not touch on this issue in the present paper.

We also need an equation for the motion of the free boundary; it is

$$
-\int_{S_{i}(t)} \sigma(t, x) \mathrm{d} \mathcal{H}^{2}(x)=\left(\kappa_{i}(t)-\beta_{i} V_{i}(t)\right)\left|S_{i}(t)\right|, \quad i=T, B, \Lambda,
$$

where $\beta_{i}$ 's are the (normalized) kinetic coefficients and (for simplicity of notation) $\left|S_{i}\right|$ stands for $\mathcal{H}^{2}\left(S_{i}\right)$, i.e. the two-dimensional Hausdorff measure of $S_{i}$. Here $\kappa_{i}$ denotes the crystalline curvature of $S_{i}$. Later, we shall see how important is the interplay of $\kappa_{i}$ 's and $\beta_{i}$ 's. Note that the kinetic coefficients here are normalized because of time normalization used in (1.3).

Condition (1.4) is in fact the Gibbs-Thomson relation. Due to the lack of smoothness of $\partial \Omega$, pointwise curvature makes no sense. Instead we use the crystalline curvatures $\kappa_{i}$ of the facets $S_{i}$ (see [GR1]). The notion of crystalline curvature was first introduced by J. Taylor [Ta] and independently by Angenent and Gurtin [AG]. We stress that the Gibbs-Thomson relation, in a similar form to [1.4), has been derived by Gurtin ([G, Chapter 8], see also [GM]) for polygonal interfaces.

These equations have to be augmented with an initial condition on $\Omega$, i.e.

$$
\Omega(0)=\Omega_{0} .
$$

In [GR1] we showed that this system (1.1)- 1.5 for $(L, R, \sigma)$ is well posed and we have local-intime existence of solutions for any admissible initial data. Namely, $L, R$ are subject to an ODE (see 
equation (2.8) and further explanations) and $\sigma$ is easily represented in terms of $L$ and $R$ (see (2.9p). However, in that paper we did not study any subtle properties of the dynamics.

Here we are interested in establishing existence of special maximal solutions (we allow for finite extinction times). Namely, we want to establish existence of self-similar solutions, i.e. such that

$$
\Omega(t)=a(t) \Omega(0)
$$

for a scale factor $a(t)$. By showing their existence we hope to gain new insight into the dynamics of (1.1) 1.5 .

At this point we mention that the existence of self-similar solutions to the anisotropic curvature flow of the form $\beta V=\kappa_{\gamma}$ is well-studied in the plane, where $\kappa_{\gamma}$ is a weighted curvature with respect to the interfacial energy $\gamma$. In fact there exists a self-similar shrinking solution for smooth, strictly convex $\gamma:[\overline{\mathrm{Ga}}],[\overline{\mathrm{GaL}}],[\overline{\mathrm{DG}}],[\overline{\mathrm{DGM}}]$. This result was extended to a planar crystalline flow in [St1], [St2] and to a crystalline flow in $\mathbb{R}^{3}$ in [PP]. Note that if $\beta \gamma=$ const, it is clear that the Wulff shape of $\gamma$ always shrinks self-similarly. The reader is referred to a review article [Gi] and a nice book [CZ] on this topic. If there is a driving force term, i.e. we deal with the equation $\beta V=\kappa_{\gamma}+C$ with some constant $C>0$, then there is a self-similar expanding solution, provided that $\beta \gamma=$ const, as shown in [So, §12]. This can be easily extended to the crystalline case. The main difference between these problems and ours is that our system may be understood as a crystalline flow coupled to a diffusion field. However, it turns out that the relation $\beta \gamma=$ const is also necessary for the existence of self-similar solutions.

Related to our work is the study of the isotropic Stefan problem undertaken in [HV]. However, the essential difference is that they do not assume the Gibbs-Thomson law at the free boundary and they work with smooth interfaces, precisely balls.

Our main goal is to establish existence of (expanding or shrinking) self-similar solutions to (1.1)-1.5. However, we will see that this is not always possible. Our result is sensitive to a choice of two sets of parameters: (a) the Wulff shape of interfacial energy density $\gamma$; (b) the kinetic coefficients $\beta_{i}$. Geometrically speaking, the crystalline curvature $\kappa_{i}$ depends on our selection of the surface energy density $\gamma$, hence on the Wulff shape $W_{\gamma}$. We note that $W_{\gamma}$ is a cylinder with constant crystalline curvature equal to -2 (see $\$ 2$ for more details). We can now give a rough indication of this dependence. We mentioned above that the evolution of $\Omega$ is in fact a system of ordinary differential equations (ODE's) for $L$ and $R$; see $(2.8)$. However, when we try to reduce this system to a single equation for the scale factor $a$ in 1.6 it turns out that this is possible only for special values of the aspect ratio

$$
\frac{L}{R}=\rho_{0},
$$

which must be equal to the quotient of the kinetic coefficients $\beta_{\Lambda} / \beta_{T}=\rho_{0}$ (see Proposition 4.2). More precisely, $\rho_{0}$ is a positive zero of a function which is not given in an explicit way. Our main focus is then on studying this function and proving that it has at least one positive root; this is done in $\S 4$.

Our work requires some preparations, made in $\S 2$, where we also explain the notation. What is more important, we present the structure of the solutions of $(1.1)-(1.5)$ and their behavior under scaling, which is an important ingredient of our argument.

We also show in $\S 3$ that the only steady state of $(1.1)-(1.5)$ is a properly scaled Wulff shape, with the scale factor depending upon $\sigma^{\infty}$. We also explain how the signs of the speeds $V_{i}$ depend on $\sigma^{\infty}$ and the curvatures $\kappa_{i}, i=T, B, \Lambda$. 


\section{Preliminaries}

In this section we set up the problem. We present our assumptions and the known results. We also recall the structure of solutions and exhibit a scaling law.

\subsection{The set up}

As in the physics literature (see [Ne], [YSF]), our evolving crystal $\Omega(t)$ is assumed to be a right cylinder,

$$
\Omega(t)=\left\{\left(x, x_{2}, x_{3}\right) \in \mathbb{R}^{3}: x_{1}^{2}+x_{2}^{2} \leqslant R^{2}(t),\left|x_{3}\right| \leqslant L(t)\right\} .
$$

In other words, we only have to know $R(t)$ and $L(t)$ to describe its evolution. The following subsets of $\partial \Omega(t)$ will be called facets:

$$
\begin{aligned}
& S_{\Lambda}=\left\{x \in \partial \Omega(t): x_{1}^{2}+x_{2}^{2}=R^{2}\right\} \\
& S_{T}=\left\{x \in \partial \Omega(t): x_{3}=L\right\} \\
& S_{B}=\left\{x \in \partial \Omega(t): x_{3}=-L\right\}
\end{aligned}
$$

these are the lateral side, top and bottom respectively. We also set $I=\{\Lambda, T, B\}$. We shall specify the initial data $\Omega(0)=\Omega_{0}$. We denote by $V_{i}$ the speed of the facet $S_{i}, i \in I$, in the direction of $\mathbf{n}$, the outer normal to $\partial \Omega(t)$.

We explicitly assume that $\sigma$ enjoys the symmetry of $\Omega(t)$, i.e. $\sigma$ is axisymmetric and symmetric with respect to the plane $x_{3}=0$ :

$$
\sigma=\bar{\sigma}\left(\sqrt{x_{1}^{2}+x_{2}^{2}},\left|x_{3}\right|\right)
$$

We want to consider a surface energy density function $\gamma$ which is consistent with our $\Omega(t)$. We recall that $\gamma$ has to be Lipschitz continuous, convex and 1-homogeneous. To be specific we take

$$
\gamma\left(x_{1}, x_{2}, x_{3}\right)=r \gamma_{\Lambda}+\left|x_{3}\right| \gamma_{T B}, \quad \gamma_{\Lambda}, \gamma_{T B}>0
$$

where $r^{2}=x_{1}^{2}+x_{2}^{2}$ and $\gamma_{\Lambda}, \gamma_{T B}$ are positive constants. Hence, its Frank diagram $F_{\gamma}$ defined as

$$
F_{\gamma}=\left\{p \in \mathbb{R}^{3}: \gamma(p) \leqslant 1\right\}
$$

consists of two right cones with a common base, which is the disk $\left\{\left(x_{1}, x_{2}, 0\right) \in \mathbb{R}^{3}: x_{1}^{2}+x_{2}^{2} \leqslant\right.$ $\left.1 / \gamma_{\Lambda}\right\}$, with the same height and with the vertices at

$$
\left(0,0, \pm 1 / \gamma_{T B}\right)
$$

Now, the Wulff shape of $\gamma$ is defined by

$$
W_{\gamma}=\left\{x \in \mathbb{R}^{3}: \forall \mathbf{n} \in \mathbb{R}^{3},|\mathbf{n}|=1, x \cdot \mathbf{n} \leqslant \gamma(\mathbf{n})\right\} .
$$

In our setting $W_{\gamma}$ is a cylinder of radius $R_{0}$ equal to $\gamma_{\Lambda}$ and half-height $L_{0}$ equal to $\gamma_{T B}$. Hence, all cylinders like $\Omega(t)$ above are admissible, in the sense that the normal $\mathbf{n}$ to the top facet (respectively: bottom, lateral surface) of $\Omega(t)$ is the normal to the top facet (respectively: bottom, lateral surface) of $W_{\gamma}$. 
We now explain how the "mean crystalline curvatures" $\kappa_{i}$ of facet $S_{i}$ may be defined. In this respect we follow [GR1]. Define the surface energy $E$ by

$$
E(S)=\int_{S} \gamma(\mathbf{n}(x)) \mathrm{d} \mathcal{H}^{2}(x) .
$$

Then we define

$$
\kappa_{i}=-\lim _{h \rightarrow 0} \frac{\Delta E}{\Delta V},
$$

where $h$ is the signed distance by which $S_{i}$ is moved in the direction of the outer normal to $S(t)$; $\Delta E$ is the resulting change of surface energy, and $\Delta V$ is the change of volume. We now recall the calculation of $\kappa_{i}$ performed in [GR1]. Namely, for $\gamma$ given by [2.1] we obtain

$$
\kappa_{T}=-\frac{2 \gamma\left(\mathbf{n}_{\Lambda}\right)}{R} \equiv \kappa_{B}, \quad \kappa_{\Lambda}=-\frac{\gamma\left(\mathbf{n}_{T}\right)}{L}-\frac{1}{R} \gamma\left(\mathbf{n}_{\Lambda}\right) .
$$

In [GR1] we pointed out a relation between $\kappa_{i}$ and the surface divergence of a selection of the Cahn-Hoffmann vector $\xi$. Here we will not pursue this topic.

We have established existence of solutions $(L, R, \sigma)$ to system (1.1)-(1.4).

Proposition 2.1 (see [GR1, Theorem 1]) There exists a unique local-in-time weak solution $(R, L, \sigma)$ to

$$
\begin{aligned}
& \Delta \sigma(t)=0 \quad \text { in } \mathbb{R}^{3} \backslash \Omega(t), \quad \lim _{|x| \rightarrow \infty} \sigma(t, x)=\sigma^{\infty}, \\
& \frac{\partial \sigma}{\partial \mathbf{n}}=V \quad \text { on } \partial \Omega(t), \\
& -\int_{S_{i}(t)} \sigma(t, x) \mathrm{d} \mathcal{H}^{2}(x)=\left(\kappa_{i}(t)-\beta_{i} V_{i}(t)\right)\left|S_{i}(t)\right|
\end{aligned}
$$

with initial condition $\Omega(0)=\Omega_{0}$, where $\Omega_{0}$ is an admissible cylinder. Moreover,

$$
R, L \in C^{1,1}([0, T)), \quad \nabla \sigma \in C^{0,1}\left([0, T) ; L^{2}\left(\mathbb{R}^{3} \backslash \Omega(t)\right)\right) .
$$

From now on, for the sake of simplicity we shall denote the unique solution to 2.2$]-2.4$ by $(\Omega, \sigma)$ instead of $(R, L, \sigma)$.

Constructing the desired solutions requires a detailed knowledge of the structure of $\sigma$. This is presented below.

\subsection{Structure of $\sigma$ and scalings}

In order to present the useful structure of $\sigma$ we have to introduce some additional objects. Namely, we need $f_{i}$ which is a unique solution to

$$
\begin{aligned}
& -\Delta f_{i}=0 \quad \text { in } \mathbb{R}^{3} \backslash \Omega, \\
& \frac{\partial f_{i}}{\partial v}=\delta_{i j} \quad \text { on } S_{j}, j \in I \text {, }
\end{aligned}
$$


such that $\nabla f_{i} \in L^{2}\left(\mathbb{R}^{3} \backslash \Omega\right)$ and $\lim _{x \rightarrow \infty} f_{i}(x)=0$ (see [GR1]), where $\Omega$ is a right cylinder in $\mathbb{R}^{3}$ given at the beginning of Section 2.1 (with time fixed). Here, $\delta_{i j}$ is the Kronecker delta and $v$ denotes the inner normal to $\partial \Omega$. For functions $f, g$ such that $\nabla f, \nabla g \in L^{2}\left(\mathbb{R}^{3} \backslash \Omega\right)$ we also define the following quantities:

$$
(f, g):=\int_{\mathbb{R}^{3} \backslash \Omega} \nabla f(x) \cdot \nabla g(x) \mathrm{d} x, \quad\|f\|^{2}:=(f, f) .
$$

Let us mention that the equation $2.5-2.6$ takes the following weak form:

$$
\int_{\mathbb{R}^{3} \backslash \Omega} \nabla f_{i}(x) \cdot \nabla h(x) \mathrm{d} x=\int_{S_{i}} h(x) \mathrm{d} \mathcal{H}^{2}(x)
$$

for all $h$ such that $\nabla h \in L^{2}\left(\mathbb{R}^{3} \backslash \Omega\right)$.

We showed in [GR1] that 2.2 - 2.4 can be reduced to the following system of ODE's:

$$
(\mathcal{A}+\mathcal{D}) \mathbf{V}=\mathbf{B}
$$

where

$$
\begin{gathered}
\mathbf{V}=\left(V_{\Lambda}, V_{T}, V_{B}\right), \quad \mathbf{B}=\left(\left|S_{\Lambda}\right|\left(\sigma^{\infty}+\kappa_{\Lambda}\right),\left|S_{T}\right|\left(\sigma^{\infty}+\kappa_{T}\right),\left|S_{B}\right|\left(\sigma^{\infty}+\kappa_{B}\right)\right), \\
\mathcal{A}=\left\{\left(f_{i}, f_{j}\right)\right\}_{i, j=\Lambda, T, B}, \quad \mathcal{D}=\operatorname{diag}\left\{\beta_{\Lambda}\left|S_{\Lambda}\right|, \beta_{T}\left|S_{T}\right|, \beta_{B}\left|S_{B}\right|\right\} .
\end{gathered}
$$

In order to explain that 2.8 is indeed an ODE we recall that

$$
V_{\Lambda}=\frac{d R}{d t}, \quad V_{T}=V_{B}=\frac{d L}{d t} .
$$

In fact, 2.8] is a system of two equations for $(R, L)$, but for the sake of compatibility with [GR1] we write it as if it were a system for three unknowns $\left(R, L_{1}, L_{2}\right)$ with $L_{1}=L_{2}$.

Moreover, $\sigma$ is given by

$$
\sigma(t)=-\sum_{i \in I} V_{i}(t) f_{i}(t)+\sigma^{\infty}
$$

The purpose of the present section is to clarify the behavior of our system under scaling of domains. Suppose we define a new variable $y$ by the formula

$$
y=a x,
$$

where $a>0$; thus $\Omega$ is transformed to $a \Omega=\tilde{\Omega}$, and $S_{i}$ goes to $a S_{i}=\tilde{S}_{i}$. If $h$ is defined on $\Omega$, then we transform it to $\tilde{h}: \tilde{\Omega} \rightarrow \mathbb{R}$ by setting

$$
\tilde{h}(y)=h(y / a) .
$$

We also define

$$
f_{i}^{a}(y)=a f_{i}(y / a), \quad i=T, B, \Lambda .
$$

The proposition below clarifies the role of $f_{i}^{a}$.

Proposition 2.2. If $f_{i}$ satisfies (2.7), then

$$
\int_{\mathbb{R}^{3} \backslash a \Omega} \nabla_{y} f_{i}^{a}(y) \nabla_{y} \tilde{h}(y) \mathrm{d} y=\int_{a S_{i}} \tilde{h}(y) \mathrm{d} \mathcal{H}^{2}(y)
$$

for all $h$ with $\nabla h \in L^{2}\left(\mathbb{R}^{3} \backslash \Omega\right)$. 
Proof. Note that $\nabla_{y} \tilde{h}(y)=\nabla_{x} h\left(\frac{y}{a}\right) \frac{D x}{D y}=\frac{1}{a} \nabla_{x} h(x)$. Then indeed

$$
\begin{aligned}
\int_{\mathbb{R}^{3} \backslash \tilde{\Omega}} \nabla_{y} f_{i}^{a}(y) \nabla_{y} \tilde{h}(y) \mathrm{d} y & =\int_{\mathbb{R}^{3} \backslash \Omega} \frac{a^{3}}{a} a \nabla_{x} f_{i}(x) \nabla_{x} h(x) \mathrm{d} x=a^{2} \int_{S_{i}} h(x) \mathrm{d} \mathcal{H}^{2}(x) \\
& =\int_{\tilde{S}_{i}} \tilde{h}(y) \mathrm{d} \mathcal{H}^{2}(y) .
\end{aligned}
$$

\section{Critical size crystals}

First, we want to determine the stationary states of 2.2 - 2.47. They will certainly depend upon $\sigma^{\infty}$. Indeed, we have the following result.

Proposition 3.1 Let $\sigma^{\infty}>0$ be given and $\gamma$ be defined by 2.1 . Then $(\Omega(t), \sigma(t))$ is a stationary solution to $2.2-(2.4)$ if and only if

$$
\Omega(0)=a W_{\gamma} \quad \text { and } \quad \sigma(t)=\sigma^{\infty},
$$

where $a=2 / \sigma^{\infty}$.

Proof. Suppose first that $\Omega(0)=a W_{\gamma}, a=2 / \sigma^{\infty}$ and $\sigma(t)=\sigma^{\infty}$. With this definition of the scale $a$, we see that the right-hand side of equation 2.8 vanishes,

$$
(\mathcal{A}+\mathcal{D}) \mathbf{V}=0 .
$$

Hence $\mathbf{V}=0$, because the matrix $\mathcal{A}+\mathcal{D}$ is nonsingular. Moreover, by 2.9$], \sigma(t) \equiv \sigma^{\infty}$. Thus $\left(\left(2 / \sigma^{\infty}\right) W_{\gamma}, \sigma^{\infty}\right)$ furnishes a solution to 2.2 - 2.4.

Conversely, if $(\Omega(t), \sigma(t))$ is a stationary solution, then 2.9 together with $V_{i} \equiv 0, i \in I$, implies that $\sigma(t)=\sigma^{\infty}$. In addition, the left-hand side of equation (2.8) vanishes, implying that $\mathbf{B}=0$. Thus, $\Omega(t)$ is of a constant crystalline curvature:

$$
\kappa_{T}=\kappa_{B}=\kappa_{\Lambda}=-\sigma^{\infty},
$$

i.e. $\Omega(t)$ is a scaled Wulff crystal. Equivalently,

$$
\frac{\gamma_{T B}}{L}=\frac{\gamma_{\Lambda}}{R}=\frac{\sigma^{\infty}}{2}
$$

and the dimensions $R, L$ are determined by $\sigma^{\infty}$.

We may now say that if $\sigma^{\infty}$ is given, then $\left(2 / \sigma^{\infty}\right) W_{\gamma}$ is of critical size. We expect that $\Omega(0)$ (not necessarily a scaled Wulff shape) containing $\left(2 / \sigma^{\infty}\right) W_{\gamma}$ will have the tendency to grow, while those $\Omega$ contained in $\left(2 / \sigma^{\infty}\right) W_{\gamma}$ will shrink. We specify this below.

Proposition 3.2 Let $\sigma^{\infty}>0$ and a solution $(\Omega(t), \sigma(t))$ to 2.2)-2.4 be given. Also assume that $V_{\Lambda}(t) \cdot V_{T}(t)>0$.

(a) If the crystalline curvatures of $\Omega(t)$ satisfy $\sigma^{\infty}+\kappa_{i}(t)>0, i \in I$, then $V_{i}(t)>0$ for all $i \in I$.

(b) If $\sigma^{\infty}+\kappa_{i}(t)<0, i \in I$, then $V_{i}(t)<0$ for all $i \in I$. 
REMARK The condition $V_{i} \cdot V_{j}>0$ looks strange, but we may not exclude the possibility that it is violated. However, in the case of self-similar motion constructed in the next section it is automatically satisfied.

Proof of Proposition 3.2. We shall prove (a) since (b) is handled in a similar way. Recall that the matrix $\mathcal{A}+\mathcal{D}$ in 2.8 is positive definite. Thus,

$$
0<\lambda_{0}|V|^{2} \leqslant((\mathcal{A}+\mathcal{D}) \mathbf{V}, \mathbf{V})=\sum_{i \in I}\left(\sigma^{\infty}+\kappa_{i}\right)\left|S_{i}\right| V_{i}
$$

Since $\sigma^{\infty}+\kappa_{i}>0$ and all terms on the right-hand side are of the same sign, we conclude that $V_{i}>0$ for $i \in\{\Lambda, T, B\}$.

\section{Existence of self-similar solutions}

We are interested in special solutions to the evolution equations that are simple yet important. We shall say that a solution $(\Omega(t), \sigma(t))$ is self-similar if at all times the region $\Omega(t)$ satisfies

$$
\Omega(t)=a(t) \Omega(0), \quad a(t) \not \equiv 1,
$$

for a real-valued function $a: \mathbb{R}_{+} \rightarrow \mathbb{R}_{+}$. We require that it is nonconstant for otherwise we would obtain a stationary solution.

We shall examine consequences of (4.1). We have to express (4.1) in terms of $R, L$ and the speeds of facets. Later we shall see that self-similar evolution exists only for some choices of the Wulff shapes $W_{\gamma}$ and kinetic coefficients $\beta_{i}, i=\Lambda, T, B$.

We note that in the case of evolving cylinder the self-similar evolution is equivalent to the constancy of the aspect ratio

$$
\frac{L(t)}{R(t)} \equiv \rho .
$$

Subsequently we shall find an equivalent characterization of 4.2 in terms of the speeds

$$
\dot{L}(t)=V_{T}(t) \equiv V_{B}(t), \quad \dot{R}=V_{\Lambda} .
$$

Proposition 4.1 Suppose that $(\Omega(t), \sigma(t))$ is a self-similar solution to 2.2 -2.4 . Then (4.2) is equivalent to

$$
\frac{V_{T}(t)}{V_{\Lambda}(t)} \equiv \rho \quad \text { for all } t \in\left[0, T_{\max }\right) \quad \text { and } \quad \frac{L(0)}{R(0)}=\rho .
$$

Proof. $\Rightarrow$ We notice that $\Omega(t)=a(t) \Omega(0)$ is equivalent to

$$
z_{i}(t)=a(t) z_{i}(0), \quad i \in\{\Lambda, T, B\}
$$

where $\left(z_{\Lambda}, z_{T}, z_{B}\right)=(R, L, L)$. After differentiation of this identity we see that

$$
V_{i}(t)=\dot{a}(t) z_{i}(0), \quad i \in\{\Lambda, T, B\} .
$$

This implies that

$$
\frac{V_{T}(t)}{V_{\Lambda}(t)}=\frac{\dot{a} L(0)}{\dot{a} R(0)}=\rho .
$$

The possibility of $\dot{a} \equiv 0$ has already been excluded in the definition. 
$\Leftarrow$ Obviously, we have

$$
z_{i}(t)=z_{i}(0)+\int_{0}^{t} V_{i}(s) \mathrm{d} s
$$

Conditions 4.3 imply

$$
\frac{L(t)}{R(t)}=\frac{L(0)+\int_{0}^{t} V_{T}(s) \mathrm{d} s}{R(0)+\int_{0}^{t} V_{\Lambda}(s) \mathrm{d} s}=\frac{\rho R(0)+\int_{0}^{t} \rho V_{\Lambda}(s) \mathrm{d} s}{R(0)+\int_{0}^{t} V_{\Lambda}(s) \mathrm{d} s}=\rho .
$$

In particular it follows from Proposition 4.1 that

$$
\frac{V_{T}(0)}{V_{\Lambda}(0)}=\rho=\frac{\frac{1}{\left|S_{T}(0)\right|} \int_{S_{T}(0)} \sigma(0, x) \mathrm{d} \mathcal{H}^{2}(x)+\kappa_{T}(0)}{\frac{1}{\left|S_{\Lambda}(0)\right|} \int_{S_{\Lambda}(0)} \sigma(0, x) \mathrm{d} \mathcal{H}^{2}(x)+\kappa_{\Lambda(0)}} \cdot \frac{\beta_{\Lambda}}{\beta_{T}}
$$

Effectively, due to representation of $\sigma$ in terms of speeds, this is an equation for the initial speeds, where the role of $\beta_{\Lambda}$ and $\beta_{T}$ has to be clarified. We shall see momentarily that the relation between $\beta_{\Lambda}$ and $\beta_{T}$ is set by another necessary condition for self-similar motion.

Proposition 4.2 Suppose that $(\Omega(t), \sigma(t))$ is a self-similar solution to 2.2 -2.4 . Then

$$
\frac{\beta_{\Lambda}}{\beta_{T}}=\frac{V_{T}(t)}{V_{\Lambda}(t)}=\rho
$$

REMARK Combining this proposition with 4.2 and the definition of the Wulff shape $W_{\gamma}$ leads us to the conclusion that the relation

$$
\beta \gamma=\mathrm{const}
$$

is a necessary condition for the existence of self-similar solutions.

Proof of Proposition 4.2 Suppose that $(\Omega(t), \sigma(t))$ is a self-similar solution. We can then express the supersaturation

$$
\sigma(t)=-\sum_{i \in\{\Lambda, T, B\}} f_{i}(t) V_{i}(t)+\sigma^{\infty}
$$

in terms of $f_{i}(\cdot) \equiv f_{i}(0, \cdot), V_{i}(0), i=T, B, \Lambda$. In $\S 2.2$ we showed that if

$$
\Omega(t)=a(t) \Omega(0),
$$

then

$$
\sigma(t)=-\sum_{i \in\{\Lambda, T, B\}} f_{i}^{a(t)} V_{i}(t)+\sigma^{\infty},
$$

where the $f_{i}^{a}$ satisfy 2.10 , i.e.

$$
f_{i}^{a(t)}(t, y) \equiv a(t) f_{i}(y / a(t))
$$

Thus, we can rewrite both sides of

$$
\int_{S_{i}(t)} \sigma(t, x) \mathrm{d} \mathcal{H}^{2}(x)=\left(-\kappa_{i}(t)+\beta_{i} V_{i}(t)\right)\left|S_{i}(t)\right|
$$


in the following way. First,

$$
\begin{aligned}
\text { LHS } & =\int_{S_{i}(t)}\left(-\sum_{j \in I} V_{j}(t) f_{j}^{a(t)}+\sigma^{\infty}\right) \mathrm{d} \mathcal{H}^{2} \\
& =-\dot{a}(t) \int_{S_{i}(t)} \sum_{j \in I} z_{j}(0) f_{j}^{a(t)} \mathrm{d} \mathcal{H}^{2}+\sigma^{\infty}\left|S_{i}(t)\right| \\
& =-\frac{\dot{a}(t)}{\dot{a}(0)} \sum_{j \in I} \int_{a(t) S_{i}(0)} \dot{a}(0) z_{j}(0) a(t) f_{j}\left(\frac{y}{a(t)}\right) \mathrm{d} \mathcal{H}^{2}(y)+\sigma^{\infty} a^{2}(t)\left|S_{i}(0)\right| .
\end{aligned}
$$

We change variable $x=y / a$ to obtain

$$
\begin{aligned}
\text { LHS } & =-\frac{\dot{a}(t)}{\dot{a}(0)} \sum_{j \in I} a^{3}(t) \int_{S_{i}(0)} V_{j}(0) f_{j}(x) \mathrm{d} \mathcal{H}^{2}(x)+\sigma^{\infty} a^{2}(t)\left|S_{i}(0)\right| \\
& =\frac{\dot{a}(t) a^{3}(t)}{\dot{a}(0)}\left(\int_{S_{i}(0)} \sigma(0, x) \mathrm{d} \mathcal{H}^{2}(x)-\sigma^{\infty}\left|S_{i}(0)\right|\right)+\sigma^{\infty} a^{2}(t)\left|S_{i}(0)\right| \\
& =\frac{\dot{a}(t) a^{3}(t)}{\dot{a}(0)}\left|S_{i}(0)\right|\left(\beta_{i} V_{i}(0)-\kappa_{i}(0)-\sigma^{\infty}\right)+\sigma^{\infty} a^{2}(t)\left|S_{i}(0)\right|
\end{aligned}
$$

We now calculate the RHS of 4.5):

$$
\begin{aligned}
\mathrm{RHS} & =\int_{a S_{i}(0)}-\frac{\kappa_{i}(0)}{a(t)} \mathrm{d} \mathcal{H}^{2}(y)+\beta_{i} \dot{a}(t) a^{2}(t)\left|S_{i}(0)\right| z_{i}(0) \\
& =-a(t) \kappa_{i}(0)\left|S_{i}(0)\right|+\beta_{i} \dot{a}(t) a^{2}(t)\left|S_{i}(0)\right| z_{i}(0) .
\end{aligned}
$$

After dividing by $\left|S_{i}(0)\right|$ we see that

$$
\sigma^{\infty} a^{2}(t)+\frac{\dot{a}(t)}{\dot{a}(0)} a^{3}(t)\left(-\kappa_{i}(0)-\sigma^{\infty}+\beta_{i} V_{i}(0)\right)=-\kappa_{i}(0) a(t)+\beta_{i} \dot{a}(t) a^{2}(t) z_{i}(0) .
$$

Further simple manipulations based on (4.5) yield

$$
\dot{a}(t) a^{2}(t)\left(\left(-\kappa_{i}(0)-\sigma^{\infty}\right) a(t)+\beta_{i} V_{i}(0)(a(t)-1)\right)=-\dot{a}(0)\left(\sigma^{\infty} a^{2}(t)+\kappa_{i}(0) a(t)\right) .
$$

If we assume that $\Omega(0)=W_{\gamma}$, then the formula for crystalline curvatures yields

$$
\kappa_{i}(0)=\kappa(\equiv-2), \quad i \in\{\Lambda, B, T\} .
$$

If we plug this result into the equations above, we obtain

$$
\dot{a}(t)=\frac{\dot{a}(0)\left(\sigma^{\infty} a(t)+\kappa\right)}{a(t)\left(\left(\sigma^{\infty}+\kappa\right) a(t)+\beta_{i} V_{i}(0)(1-a(t))\right)}, \quad i \in\{\Lambda, B, T\} .
$$

Of course, we have to assume that $\sigma^{\infty}+\kappa \neq 0$, for otherwise we would have a stationary solution. If the left-hand side is to be independent of $i$, then we conclude that

$$
\beta_{T} V_{T}(t)=\beta_{\Lambda} V_{\Lambda}(t),
$$

as desired. 
On the way, we established the equation that $a(t)$ must fulfill, namely $(4.6)$. This equation has to be augmented with an initial condition, i.e.

$$
a(0)=1
$$

and we have to supply $\dot{a}(0)$ calculated from (4.5). Namely, (4.5) may be rewritten as

$$
\sum_{j \in I} V_{j}(0)\left(f_{i}, f_{j}\right)+\beta_{i}\left|S_{i}(0)\right| V_{i}(0)=\left(\sigma^{\infty}+\kappa_{i}(0)\right)\left|S_{i}(0)\right| .
$$

Before we proceed, we make a simplifying assumption. Due to the scaling formula of $\S 2.2$, without loss of generality we may assume

$$
L(0)=\rho \quad \text { and } \quad R(0)=1 .
$$

If we use this simplification, Cramer's formula applied to 2.8 yields

$$
\frac{V_{T}(0)}{V_{\Lambda}(0)}=\frac{\left\|f_{\Lambda}\right\|^{2}+4 \beta_{\Lambda} \pi \rho-8 \rho\left(f_{\Lambda}, f_{T}\right)}{4 \rho\left(f_{T}, f_{T}+f_{B}\right)+4 \pi \rho^{2} \beta_{T}-2\left(f_{\Lambda}, f_{T}\right)} .
$$

Combining this with

$$
\frac{V_{T}(0)}{V_{\Lambda}(0)}=\rho,
$$

we obtain an equation for $\rho$, where we also use $\beta_{\Lambda} / \beta_{T}=\rho$.

After some simple algebraic manipulations, the equation for $\rho$ reduces to

$$
4 \rho^{2}\left(f_{T}, f_{T}+f_{B}\right)+6 \rho\left(f_{T}, f_{\Lambda}\right)=\left\|f_{\Lambda}\right\|^{2} .
$$

We notice that $\beta_{\Lambda}, \beta_{T}$ miraculously cancel out.

We stress that $f_{T}, f_{B}, f_{\Lambda}$ depend only on $\rho$ and they are independent of $\sigma^{\infty}$.

This is the equation for the constant-in-time proportions of the self-similar evolving cylinder. It guarantees that

$$
\frac{L(0)}{R(0)}=\frac{V_{T}(0)}{V_{\Lambda}(0)}=\frac{\beta_{\Lambda}}{\beta_{T}}=\rho .
$$

We will show that (4.8) has at least one solution. We first calculate the behavior of both sides of (4.8) for large and small $\rho$. This will be done in a series of lemmata. We do not try to give optimal estimates here. We content ourselves with ones sufficient for showing the existence of solutions.

LEMmA 4.3 There exists a universal constant $C_{1}$ independent of $\rho$ such that

$$
\left\|f_{\Lambda}\right\| \leqslant C_{1} \rho^{1 / 2}\left(1+\rho^{1 / 3}\right), \quad \forall \rho \in \mathbb{R}_{+},
$$

and $\left\|f_{\Lambda}\right\| \leqslant C_{1} \rho^{3 / 4}$ for small $\rho$.

Proof. Choose a cut-off function $\eta:[0, \infty) \rightarrow[0,1]$ such that $\eta(1)=1$ and

$$
\eta(r)= \begin{cases}(R+l-r) / l, & r \in[R, R+l], \\ 0, & r>R+l, \\ 1, & r \in[0, R) .\end{cases}
$$


Of course $R=1$ due to our scaling, but for the sake of clarity we shall exhibit it anyway. Note that

$$
\left\|f_{\Lambda}\right\|^{2}=\int_{S_{\Lambda}} f_{\Lambda}(x) \mathrm{d} \mathcal{H}^{2}(x)=2 \int_{0}^{2 \pi} \int_{0}^{\rho} f_{\Lambda}(x) R \mathrm{~d} x_{3} \mathrm{~d} \theta
$$

where $\left(r, \theta, x_{3}\right)$ are cylindrical coordinates.

Set $D=(B(0, R+l) \backslash B(0, R)) \times(-\rho, \rho)$. Then due to the definition of $\eta$ we have

$$
\begin{aligned}
\left\|f_{\Lambda}\right\|^{2} & =2 \int_{0}^{2 \pi} \int_{0}^{\rho}\left[f_{\Lambda}(x) R \eta(R)-f_{\Lambda}(x)(R+l) \eta(R+l)\right] \mathrm{d} x_{3} \mathrm{~d} \theta \\
& =-2 \int_{0}^{2 \pi} \int_{0}^{\rho} \int_{R}^{R+l} \frac{\partial}{\partial r}\left(\eta(r) r f_{\Lambda}(x)\right) \mathrm{d} x_{3} \mathrm{~d} \theta \mathrm{d} r \\
& =-\int_{D}\left(\frac{\partial}{\partial r} \eta f_{\Lambda}+\eta \frac{\partial f_{\Lambda}}{\partial r}+\frac{\eta}{r} f_{\Lambda}\right) \mathrm{d} x
\end{aligned}
$$

We notice that $|D|=2 \rho \pi(2 R+l) l$ and $\eta / r \leqslant 1$ for $r \geqslant R=1$. Now, by the Hölder inequality,

$$
\left\|f_{\Lambda}\right\|^{2} \leqslant\left(1+\frac{1}{l}\right)|D|^{5 / 6}\left(\int_{D} f_{\Lambda}^{6}\right)^{1 / 6}+|D|^{1 / 2}\left(\int_{D}\left|\nabla f_{\Lambda}\right|^{2}\right)^{1 / 2} .
$$

The first integral may be estimated by the Sobolev inequality for unbounded domains (see e.g. [HK, Theorem 5]), i.e. we obtain

$$
\left\|f_{\Lambda}\right\| \leqslant c \rho^{5 / 6}\left(l^{5 / 6}+l^{-1 / 6}\right)(1+l)^{5 / 6}+c \rho^{1 / 2} l^{1 / 2}(1+l)^{1 / 2},
$$

where $c$ is a constant. When we choose $l=1$, then

$$
\left\|f_{\Lambda}\right\| \leqslant c \rho^{1 / 2}\left(1+\rho^{1 / 3}\right), \quad \forall \rho \in \mathbb{R}_{+} .
$$

For small $\rho$ it is advantageous to balance all the terms in the estimate; for this reason we take $l=\rho^{1 / 2}$. Hence,

$$
\left\|f_{\Lambda}\right\| \leqslant c \rho^{5 / 6-1 / 12}+c \rho^{1 / 2+1 / 4}=: C_{1} \rho^{3 / 4}
$$

for sufficiently small $\rho>0$, as desired.

A similar reasoning leads us to

LEMMA 4.4 There exists a constant $C_{2}$ independent of $\rho$ such that

$$
\left\|f_{T}\right\| \leqslant C_{2} .
$$

We also need good lower bounds on $\left\|f_{\Lambda}\right\|$.

LEMMA 4.5 There exists $C_{3}>0$ such that for all sufficiently small $\rho$ the following estimate holds:

$$
\left\|f_{\Lambda}\right\| \geqslant C_{3} \rho^{5 / 6} \text {. }
$$

Proof. We shall exploit the fact that for any function $\eta$ with $\nabla \eta \in L^{2}$ and equal to 1 on $S_{\Lambda}$, we have

$$
4 \pi R \rho=\int_{S_{\Lambda}} 1 \mathrm{~d} \mathcal{H}^{2}=\int_{\mathbb{R}^{3} \backslash \Omega} \nabla \eta \cdot \nabla f_{\Lambda} \leqslant\left\|f_{\Lambda}\right\| \cdot\|\eta\| .
$$


We take $\eta=\psi(r) \varphi\left(x_{3}\right)$, where $r^{2}=x_{1}^{2}+x_{2}^{2}$ and $\varphi(z)$ is even. Specifically, we choose

$$
\begin{aligned}
& \psi(r)= \begin{cases}1, & r \in[0, R], \\
(R+\ell-r) / \ell, & r \in(R, R+\ell], \\
0, & r \geqslant R+\ell,\end{cases} \\
& \varphi(z)= \begin{cases}1, & z \in[0, \rho], \\
(\rho+\lambda-z) / L, & z \in(\rho, \rho+\lambda], \\
0, & z>\rho+\lambda,\end{cases}
\end{aligned}
$$

where $\ell, \lambda>0$ will be picked later. Obviously

$$
\nabla \eta=\psi_{r} \varphi \cdot \vec{e}_{r}+\psi \varphi_{x_{3}} \vec{e}_{x_{3}},
$$

where $\vec{e}_{r}=\left(x_{1}, x_{2}, 0\right) / r, \vec{e}_{x_{3}}=(0,0,1)$ and $|\nabla \eta|^{2}=\psi_{r}^{2} \varphi^{2}+\psi^{2} \varphi_{x_{3}}^{2}$. We now set

$$
\begin{aligned}
& D=[B(0, R+\ell) \backslash B(0, R)] \times[-\rho, \rho], \\
& P=B(0, R+\ell) \times[\rho, \lambda+\rho] \cup B(0, R+\ell) \times[-\lambda-\rho,-\rho], \\
& X=[B(0, R+\ell) \backslash B(0, R)] \times([-\lambda-\rho,-\rho] \cup[\rho, \rho+\lambda]) .
\end{aligned}
$$

With these definitions,

$$
\int_{\mathbb{R}^{3} \backslash \Omega}|\nabla \eta|^{2} \mathrm{~d} x=\int_{D} \psi_{r}^{2} \varphi^{2} \mathrm{~d} x+\int_{P} \psi^{2} \varphi_{x_{3}}^{2} \mathrm{~d} x+\int_{X} \psi_{r}^{2} \varphi^{2} \mathrm{~d} x .
$$

We shall treat each term separately. On $D$ we clearly have $\psi_{r}=-\ell^{-1}$, hence

$$
\int_{D} \varphi^{2} \psi_{r}^{2} \mathrm{~d} x \leqslant \frac{1}{\ell^{2}}|D|=\frac{2 \pi \rho}{\ell}(2 R+\ell)
$$

On $P$ we have $\left|\varphi_{x_{3}}\right|=1 / \lambda$ and

$$
\int_{P} \varphi_{x_{3}}^{2} \psi^{2} \mathrm{~d} x \leqslant \frac{\pi}{\lambda^{2}}(R+\ell)^{2},
$$

and finally we calculate the integral over $X$ :

$$
\int_{X} \psi_{r}^{2} \varphi^{2} \mathrm{~d} x \leqslant \frac{2 \pi}{\ell} \lambda(2 R+\ell)
$$

Combining those results we arrive at

$$
\|\eta\| \leqslant c\left(\frac{3}{\ell}(1+\ell)+\frac{(1+\ell)^{2}}{\lambda}+\frac{\lambda}{\ell}(1+\ell)\right)^{1 / 2} .
$$

Again, we want to balance all the terms, so we put $\ell=\rho^{\alpha}, \lambda=\rho^{-\beta}, \alpha, \beta>0$. The constraint of equal powers of all terms yields $\beta=1 / 3, \alpha=2 / 3$, and finally

$$
\|\eta\| \leqslant c \rho^{1 / 6} \text {. }
$$

Hence $\left\|f_{\Lambda}\right\| \geqslant C_{3} \rho^{5 / 6}$ for all $\rho>0$. 
The argument used above also yields

Lemma 4.6 There exists $C_{4}>0$ such that for all $\rho>0$,

$$
\left\|f_{T}\right\| \geqslant C_{4}
$$

i.e. $\left\|f_{T}\right\|$ is bounded below by a positive constant.

We are now ready to study equation 4.8.

THEOREM 4.7 Equation (4.8) has at least one positive solution $\rho_{0}$.

Proof. Both sides of (4.8) are continuous functions of $\rho$. We shall estimate the growth of both sides at $\infty$ and near 0 .

For $\rho>1$ due to Lemmas 4.3 and 4.6 we have

$$
\text { RHS } \leqslant C_{U} \rho^{5 / 6}, \quad \text { LHS } \geqslant 4 \rho^{2}\left\|f_{T}\right\| \geqslant C_{L} \rho^{2},
$$

where we also used the positivity of $\left(f_{T}, f_{B}\right)$ and $\left(f_{T}, f_{\Lambda}\right)$. Hence, LHS $>$ RHS for sufficiently large $\rho$.

Now, for small $\rho>0$, Lemmas $4.5,4.3$ and 4.4 yield

$$
\text { RHS } \geqslant c_{l} \rho^{5 / 3}, \quad \text { LHS } \leqslant c\left(\rho^{2}+\rho \rho^{3 / 4}\right) .
$$

It is obvious that

$$
\text { LHS }<\text { RHS for sufficiently small } \rho>0 .
$$

Hence, for some $\rho_{0}>0$ both sides of 4.8 must be equal, because they are continuous functions of $\rho$.

So far, we have determined the proportions of the Wulff shape and the proportions of the kinetic coefficients $\rho_{0}=\beta_{\Lambda} / \beta_{T}$. The number $\rho_{0}$ is special and may not be unique. We are now ready to establish the existence of a self-similar evolution.

THEOREM 4.8 Suppose that $\rho_{0}$ is a solution of 4.8 and $\Omega(0)$ is the Wulff shape of $\gamma$, i.e. $\Omega(0)=$ $W_{\gamma}$ and $L_{0} / R_{0}=L(0) / R(0)=\rho_{0}$. Assume that

$$
\frac{\beta_{\Lambda}}{\beta_{T}}=\rho_{0} .
$$

Then the evolution of $\Omega(0)$ is self-similar.

Proof. We have to show that $\Omega(t)=a(t) \Omega(0)$. We have already shown in the course of proof of Proposition 4.2 that $a(t)$ satisfies the ODE 4.6,

$$
\begin{aligned}
\dot{a} & =\frac{\dot{a}(0)\left(\sigma^{\infty} a(t)+\kappa\right)}{a(t)\left(\left(\sigma^{\infty}+\kappa\right) a(t)+\beta_{T} V_{T}(0)(1-a(t))\right)}, \\
a(0) & =1,
\end{aligned}
$$

where $\beta_{T} V_{T}(0)=\beta_{\Lambda} V_{\Lambda}(0)$ by our choice of $\rho_{0}$ and $\kappa=\kappa_{T}=\kappa_{\Lambda}$. We recall that $\kappa$ denotes the mean crystalline curvature of $\Omega(t)$ at time $t=0$, i.e. when $\Omega(0)=W_{\gamma}$. This is a constant in 4.9. We also stress that $\sigma^{\infty}$ is a constant parameter of the problem (see equation $(1.2)$ ). 
Similarly, we know that

$$
\dot{a}(0)=\frac{V_{\Lambda}(0)}{R(0)}=\frac{V_{T}(0)}{L(0)} .
$$

Equation (4.9) has a unique solution, because the right-hand side of (4.9) is Lipschitz continuous with respect to $a$ and the denominator never vanishes. This is so due to

$$
\begin{aligned}
\left(\sigma^{\infty}+\kappa\right) a(t)-\beta_{T} V_{T}(0) a(t)+\beta_{T} V_{T}(0) & =a(t)\left(\sigma^{\infty}+\kappa-\beta_{T} V_{T}(0)\right)+\beta_{T} V_{T}(0) \\
& =a(t) \int_{S_{T}(0)} \sum_{i \in I} V_{i}(0) f_{i} \mathrm{~d} \mathcal{H}^{2}+\beta_{T} V_{T}(0) \\
& \neq 0
\end{aligned}
$$

as long as $\sigma^{\infty} \neq-\kappa$ and $V_{i}$ 's have the same sign.

We have to check whether

$$
\left(V_{\Lambda}(t), V_{T}(t)\right):=\left(\dot{a}(t) z_{\Lambda}(0), \dot{a}(t) z_{T}(0)\right) \equiv \dot{a} \cdot(R(0), L(0))
$$

and

$$
\sigma(t):=-\sum_{i \in I} \dot{a}(t) z_{i} f_{i}^{a(t)}+\sigma^{\infty}
$$

satisfy $2.27-2.47$. Of course

$$
\Delta \sigma(t)=0 \quad \text { in } \mathbb{R}^{3} \backslash \Omega(t), \quad \lim _{|x| \rightarrow \infty} \sigma(t, x)=\sigma^{\infty},
$$

and

$$
\frac{\partial \sigma}{\partial \mathbf{n}}=V_{i} \quad \text { on } \partial \Omega(t)
$$

by the properties of $f_{i}^{a}$ (see Proposition 2.2. Moreover,

$$
\int_{S_{i}(t)} \sigma(t) \mathrm{d} \mathcal{H}^{2}=\int_{S_{i}(t)}\left(\beta_{i} V_{i}(t)-\kappa_{i}(t)\right) \mathrm{d} \mathcal{H}^{2}, \quad i=T, B, \Lambda,
$$

by the very definition of $\dot{a}(t)$.

Let us remark that if $V_{i}>0$, then $a(t)$ is defined for all $t \geqslant 0$. On the other hand, if $\Omega(t)$ shrinks, i.e. $V_{i}<0$, then it is easy to see from (4.9) that the solution dies out in finite time. We leave this calculation to the interested reader.

\section{Acknowledgments}

The work of the first author was partly supported by a Grant-in-Aid for Scientific Research, Nos. 14204011 and 1563408, of the Japan Society for the Promotion of Science. The second author was supported in part by KBN grant 2 P03A 042 24. Both authors enjoyed support resulting from the Polish-Japanese Intergovernmental Agreement on Cooperation in the Field of Science and Technology. 


\section{REFERENCES}

[AG] Angenent, S. B. \& Gurtin, M. E. Multiphase thermomechanics with interfacial structure 2. Evolution of an isothermal interface. Arch. Rat. Mech. Anal. 108 (1989), 323-391. Zbl 0723.73017 MR 1013461

[BNP1] Bellettini, G., Novaga, M., \& Paolini, M. Facet-breaking for three dimensional crystals evolving by mean curvature. Interfaces Free Bound. 1 (1999), 39-55. Zbl 0934.49023 MR 1865105

[BNP2] Bellettini, G., Novaga, M., \& Paolini, M. Characterization of facet-breaking for nonsmooth mean curvature flow in the convex case. Interfaces Free Bound. 3 (2001), 415-446. Zbl 0989.35127 MR 1869587

[CZ] CHOU, K.-S. \& ZHU, X. P. The Curve Shortening Problem, Chapman and Hall/CRC, Boca Raton, FL (2001). Zbl 01589771 MR 1888641

[DG] Dohmen, C. \& GIGA, Y. Selfsimilar shrinking curves for anisotropic curvature flow equations. Proc. Japan Acad. Ser. A 70 (1994), 252-255. Zbl 0815.34026 MR 1303574

[DGM] Dohmen, C., Giga, Y., \& Mizoguchi, N. Existence of selfsimilar shrinking curves for anisotropic curvature flow equations. Calc. Var. Partial Differential Equations 4 (1996), 103-119. Zbl 0847.34042 MR 1379195

[Ga] Gage, M. E. Evolving plane curves by curvature in relative geometries. Duke Math. J. 72 (1993), 441-466. Zbl 0798.53041 MR 1248680

[GaL] GaGe, M. E. \& LI, Y. Evolving plane curves by curvature in relative geometries II. Duke Math. J. 75 (1994), 79-98. Zbl 0811.53033 MR 1284816

[GiGi] Giga, M.-H. \& GigA, Y. A subdifferential interpretation of crystalline motion under nonuniform driving force. Dynamical Systems and Differential Equations (Springfield, MO, 1996), W.-X. Chen and S.-C. Hu (eds.), Southwest Missouri Univ., Vol. 1 (1998), 276-287. MR 1720610

[Gi] GigA, Y. Anisotropic curvature effects in interface dynamics. Sūgaku 52 (2000), 113-127 (English transl. in Sūgaku Exposition).

[GR1] GigA, Y. \& RYBKA, P. Quasi-static evolution of 3-D crystals grown from supersaturated vapor. Differential Integral Equations 15 (2002), 1-15. Zbl 01836159 MR 1869818

[GR2] GigA, Y. \& RyBKA, P. Berg's effect. Adv. Math. Sci. Appl. 13 (2003), 625-637.

[GoG] GondA, T. \& Gomi, H. Morphological instability of polyhedral ice crystals growing in air at low temperature. Ann. Glaciology 6 (1985), 222-224. Zbl 0787.73004

[G] Gurtin, M. Thermomechanics of Evolving Phase Boundaries in the Plane. Clarendon Press, Oxford, 1993. Zbl 0787.73004 MR 1402243

[GM] Gurtin, M. \& Matias, J. Thermomechanics and the formulation of the Stefan problem for fully faceted interfaces. Quart. Appl. Math. 53 (1995), 761-782. Zbl 0841.76086 MR 1359510

[HK] HajŁasz, P. \& KaŁamajska, A. Polynomial asymptotics and approximation of Sobolev functions. Studia Math. 113 (1995), 55-64. Zbl 0823.46036 MR 1315521

[HV] Herrero, M. A. \& Velázquez, J. J. L. On the melting of ice balls. SiaM J. Math. Anal. 28 (1997), 1-32. Zbl 0876.35137 MR 1427725

[KIO] KURODA, T., IRISAWA, T., \& OOKAWA, A. Growth of a polyhedral crystal from solution and its morphological stability. J. Crystal Growth 42 (1977), 41-46.

[Ne] Nelson, J. Growth mechanisms to explain the primary and secondary habits of snow crystals. Philos. Mag. A 81 (2001), 2337-2373.

[PP] Paolini, M. \& Pasquarelli, F. Unstable crystalline Wulff shapes in 3D. Variational Methods for Discontinuous Structures, Progr. Nonlinear Differential Equations Appl. 51, Birkhäuser, Basel (2002), 141-153. Zbl 1032.82027 
[Se] SEEgER, A. Diffusion problems associated with the growth of crystals from dilute solution. Philos. Mag. 44 (1953), 1-13.

[So] Soner, M. Motion of a set by curvature. J. Differential Equations 101 (1993), 313-372.

[St1] Stancu, A. Uniqueness of self-similar solutions for a crystalline flow. Indiana Univ. Math. J. 45 (1996), 1157-1174. Zbl 0769.35070 MR 1204331

[St2] StANCU, A. Asymptotic behaviour of solutions to a crystalline flow. Hokkaido Math. J. 27 (1998), 303-320. Zbl 0989.53042 MR 1637988

[Ta] TAYLOR, J. E. Motion of curves by crystalline curvature, including triple junctions and boundary points. Differential Geometry Partial Differential Equations on Manifolds (Los Angeles, CA, 1990), Proc. Sympos. Pure Math. 54, Amer. Math. Soc. (1993), 417-438. Zbl 0823.49028 MR 1216599

[YSF] YokoYama, E., SEKERKA, R.F., \& FurukawA, Y. Growth trajectories of disk crystals of ice growing from supercooled water. J. Phys. Chem. B 104 (2000), 65-67. 